\title{
Epidemiologische und hygienische Mitteilungen über eine
} Fleckfieberepidemie.

\author{
Von
}

Dr. Philippsthal, landsturmpflichtiger Arzt.

Der Gang der Fleckfieberepidemie in Focsani und ihre Wirkung auf die Bevölkerung ist vom 1. März 1917 bis 28. Februar 1918 beobachtet und registriert worden.

Bis zum 1. Juli 1917 sind die Fälle nur klinisch diagnostiziert; von diesem Termin an wurde auch die serologische Untersuchung nach WeilFelix zunächst in vereinzelten Fällen vorgenommen und von Ende September an im Laboratorium des Korps in allen Fällen durchgeführt. In zweifelhaften Fällen entschied der klinische Befund.

Vor dem Krieg hat es Typhus exanthematicus nach übereinstimmendem Urteil der rumänischen Ärzte in Focsani nicht gegeben. Die Seuche kam mit dem Krieg über das Land, wahrscheinlich mit den ersten rumänischen oder russiscken Gefangenen. Die ersten Fälle sind aber erst von deutschen Ärzten in Focsani festgestellt, wohl deshalb, weil den Rumänen das Krankheitsbild des Typhus exanthematicus nicht bekannt war. Die Krankheit nahm nach und nach zur Zeit der deutschen Besetzung den Charakter einer Epidemie an.

Ein so wichtiges Ereignis wie eine Fleckfieberepidemie läßt sich nicht getrennt von der sozialen und wirtschaftlicken Lage der Zeit betrachten. Sie ist ein Ausdruck für die schwere Not, die eine Bevölkerung ergriffen hat. Die Analyse der Notlage bedeutet deshalb eine Erklärung der Epidemie. Der Krieg ist nicht nur eine Kampfhandlung zwischen zwei Fronten, sondern auch ein Eingriff, der das gesellschaftliche. Leben der beteiligten Völker in allen seinen Äußerungen stört. Die Bevölkerung, die den Fronten am nächsten wohnt, wird am schwersten getroffen. 
Die hygienische Komfortbreite des einzelnen wird eingeengt (Wohnungsnot); nur das Nahrungsmittelminimum wird gewährt (Narrungsmittelnot); dazu kommt die seelische Belastung.

Viele werden aus ihrem Besitz vertrieben, in ein anderes Milieu geworfen (Flïchtlinge). Auf diese Verschlechterung der Lebensbedingungen reagiert der menschliche Organismus mit Störungen, die zur Krankheit und, wenn sie einen infektiösen Charakter trägt, leicht zur Epidenie ausarten.

Focsani hatte kurz vor der deutsclen Okkupation nach Angabe des Einwohnermeldeamtes 21759 Einwolner. Als die deutschen Truppen in der Näle von $F$. feste Stellungen bezogen, wurden die Bewolner der Dörfer des Frontgebietes nach $\mathrm{F}$. gedrängt. Damals siedelten sich noch 8650 Fiüchtlinge in F. an. Außerdem wurden Stäbe und Formationen in F. einquartiert; die Zahl schwankt, weshajb genaue Angaben nicht gemacht werden können. Jedenfalls wurde durch die Truppen der Wohnungsraum der Zivilbevölkerung erlieblich eingeschränkt.

So wurde eine Stadt, die zur Aufnahme von etwa 23000 Personen eingerichtet war, mit etwa 34000 belegt.

Zur Überbelegung trat die Hungersnot. Das fliehende rumänische Heer hatte große Nahrungsmittelmengen nach der Moldau verschleppt. Die Russen hatten viel Getreide, um es nicht in deutsche Hände fallen zu lassen, ihrem Brauche gemäß durch Feuer vernichtet. Der Bestand an Nahrungsmitteln in der Walachei, besonders in Focsani, war deshalb gering. Die Flüchtlinge, die sich in $\mathrm{F}$. ansiedelten, hatten bei ihrer eiligen Flucht nur das Notwendigste mitgenommen. Der Vorrat der Städter war bald verbraucht. Bis die Regulierung durch die deutsche Verwaltung einsetzte, verging einige Zeit, weil erst die Transportverhältnisse eingerichtet werden mußten. Und dann konnte auch nur die Erhaltungsquote gewährt werden.

Innerhalb der aus den Fugen geratenen Stadtgemeinde begann die Fleckfieberlaus, vom Kriege dorthin getragen, ibr verderbliches Werk.

Vom 1: März 1917 bis 28. Februar 1918 sind in Focsani 671 Fälle von Typhus exantlematicus zur Meldung gekommen. Bei jedem Versuch läßt sich zwischen den Fällen ein lokaler Kontakt nachweisen. Die Epidemiepause überdakert niemals die Inktbationszeit. Die Epidemiekurve bewegt sich wellenförmig. Die erste Welle setzt im Februar schleichend ein, steigt allmählich bis Ende April und stürmisch im Juni hoch an, um im September harmlos zu verebben.

Die Nachwelle beginnt langsam im Oktober, hebt sich bis zum Januar, ohne je die gefährliche Höhe der ersten Welle erreicht zu haben, und fällt im Februar. Es besteht eine Kontinuität beidex Epidemien; 
zwischen Sommer- und Winterepidemie wurden im September 8 leichte Fälle von Typhus exantrematicus festgestellt.

Die Mortalität an Fleckfieber beträgt im Beobachtungsjahr 177 Fälle. Uavon entfallen auf die erste Fleckfieberperiode 145, anf die zweite Fleckfieberperiode 32 Fälle.

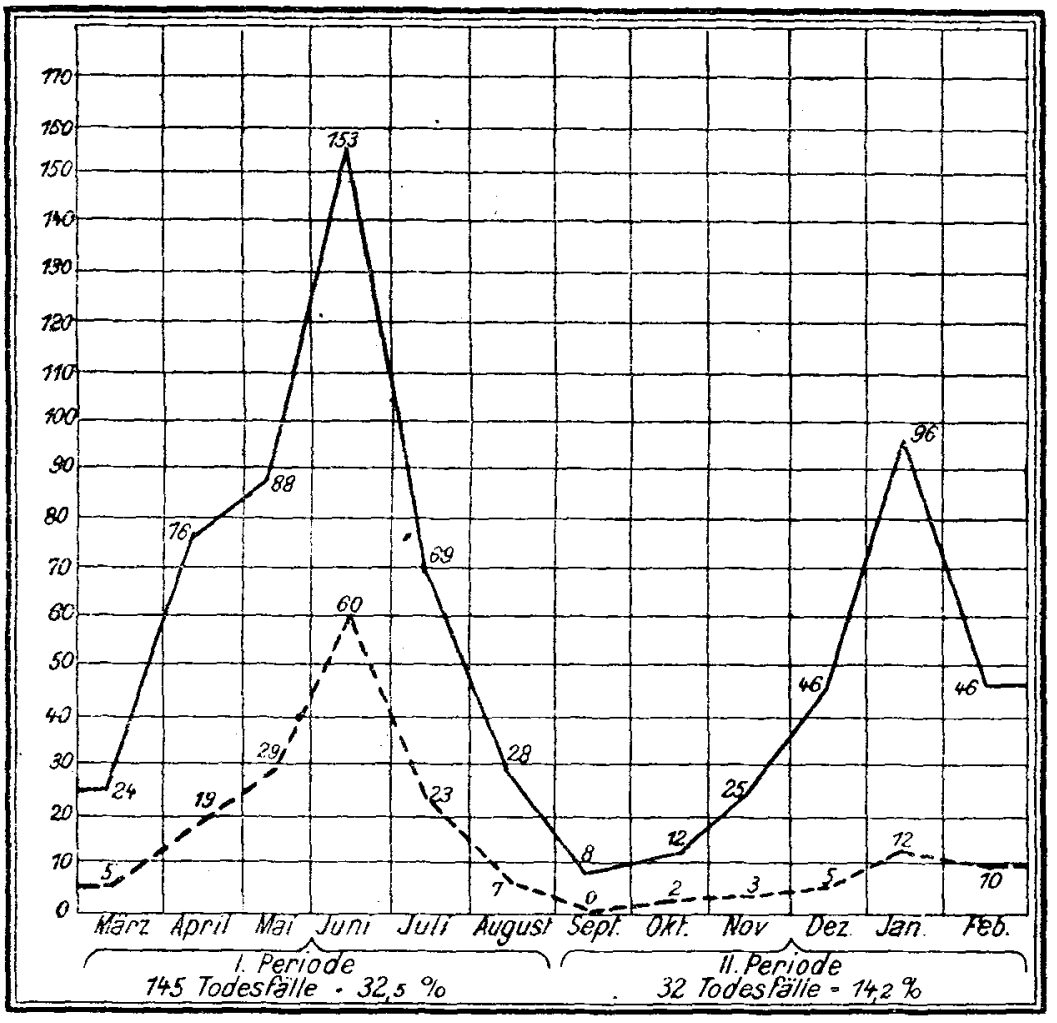

Fleckfieber-Morbidität.

Fleckfieber-Mortalität.

Fig. 1.

Dic Gesamtepidemie hat eine Sterblichkeitsziffer von 26.3 Prozent. 1)ie Differenz im prognostischen (harakter beider Perioden geht aus einer Gegenuberstellung lervor:

Die erste Periode hat 32.5 Prozent, die zweite $14 \cdot 2$ Prozent Mortalität.

Die Sterblichkeitskurve der ersten Periode korrespondiert mit der Krankheitskurve der gleichen Zeit, wälirend die zweite Periode keine, Korrespondenz im Verhältnis von Krankheit und Sterblichkeit aufweist.

Die Krankheitsfälle, die im Februar 1917 bekannt wurden, betrafen Flüchtlinge: die Krankheitsherde blieben stets in den Flüchtlingsquartieren; 
von dort bewegte sich die Seuche in die Stadtbevölkerung. Eine statistische Untersuchung der Epidemie ergibt, da $B$ die Fleckfieberepidemie in Focsani im wesentlichen eine Durchseuchung der Flüchtlinge gewesen ist.

Es erkranken in der 1. Periode

\begin{tabular}{cc} 
Städter & Flüchtlinge \\
208 & 237 \\
140 & 86 \\
\hline 348 & 323 \\
\hline $24 \cdot 5$ Prozent & $94=36 \cdot 7$ Prozent \\
$=15 \cdot 0 \quad,$, & $11=12 \cdot 7 \quad,$, \\
\hline $20 \cdot 7$ Prozent & $105=32 \cdot 5$ Prozent \\
\hline
\end{tabular}

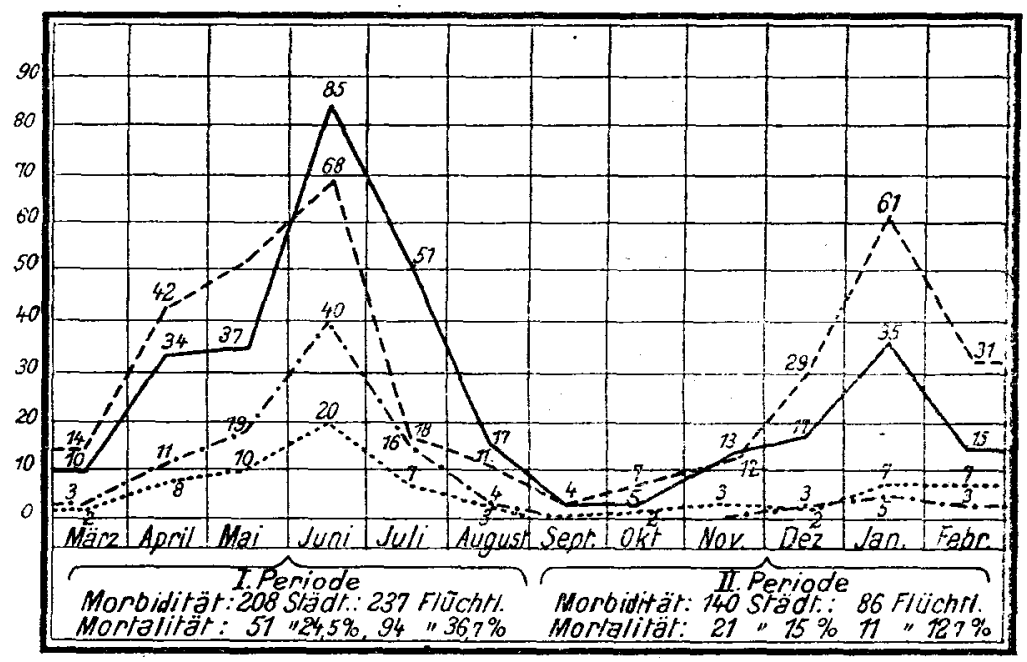

Morbidität der flüchtlinge

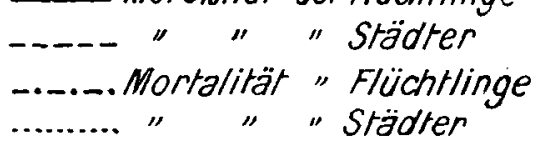

Fig. 2.

Erst die relativen Zahlen geben ein klares Bild von der Beteiligung der Flüchtlinge an der Seuche.

Es erkranken im monatlichen Durchschnitt:

Von 1000 Städtern Von 1000 Flüchtlinger:

$\begin{array}{rrrr}\text { in der } 1 \text {. Periode . . . } & 1 \cdot 6 & 14 \cdot 6 \\ . . .22 . \quad, \quad . . & 1 \cdot 2 & 4 \cdot 2\end{array}$


Es sterben im monatlichen Durchschnitt:

$\begin{array}{ccccc} & & & \text { Von } 1000 \text { Städtern } & \text { Von } 1000 \text { Flüch tlingen } \\ \text { in der } 1 \text {. Periode . . . . } & 0.3 & 1.9 \\ \therefore \quad ., 2 . & \text {. } & \text {. . . . } & 0.02 & 0.56\end{array}$

Der Mensch, der mit dem Fleckfiebererreger infiziert wird, erkrankt an Fleckfieber ohne Rücksicht auf das Alter. Alle Lebensalter werden erfaßt. Daß das Säuglingsalter und das Alter der kleinen Kinder in der Statistik geringer vertreten ist, liegt an der Schwierigkeit der Diagnose. Klinisch tritt bei jungen Individuen die Krankheit meist so leicht auf, daß Fleckfieber nicht vermutet wird. Abortivfälle sind häufig, die zufällig durch die serologische Reaktion gesichert werden, oft genug aber der Diagnose entgehen. Mithin besteht kein Zweifel, daB Säuglinge und kleine Kinder häufiger an Fleckfieber erkranken als die Statistik zeigt.

In der 1. Periode erkianken:

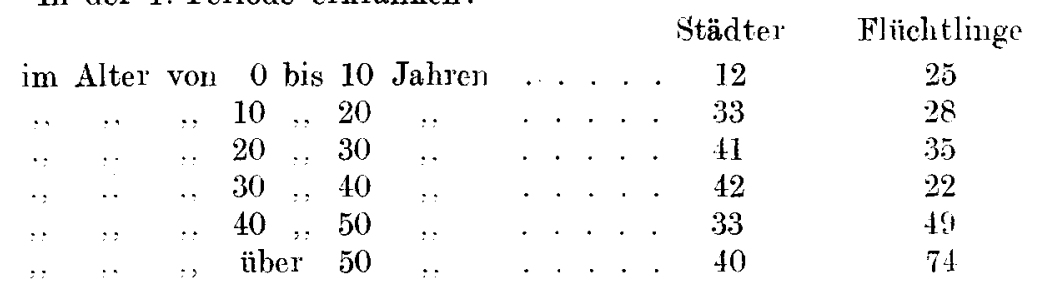

In der 2. Periode erkranken:

Städter Flüchtlinge

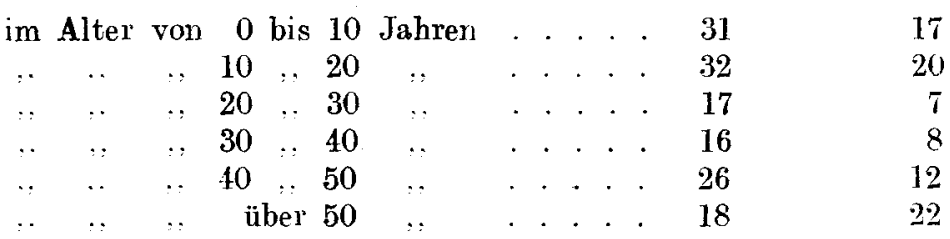

Die Mortalitätsziffern, nach Lebensaltern geordnet, lassen das Gesetz, daB die Malignität des Typhus exanthematicus mit dem Alter wächst, klar erkennen.

In der 1. Periode sterben:

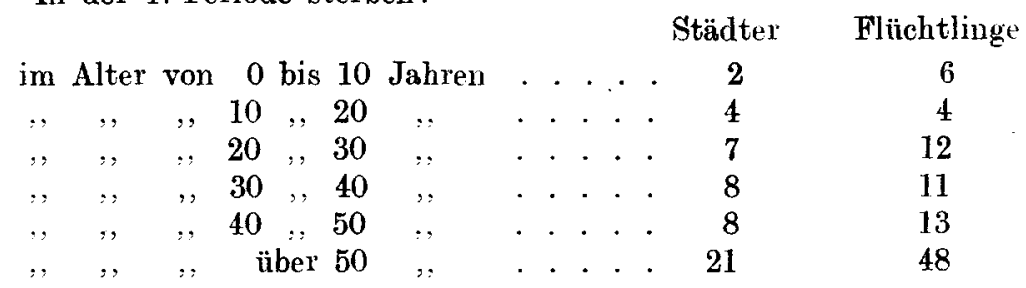


In der 2. Periode sterben:

Städter Flüchtlinge

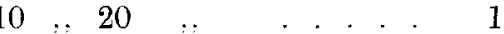

$\begin{array}{llllll}. & . . & . . & 20 & , & 30\end{array}$

$30 \quad: \quad 40$

$40,, 50$

über 50
0

2

3

7

7
2

3

3

I. Periode.

II. Periode.

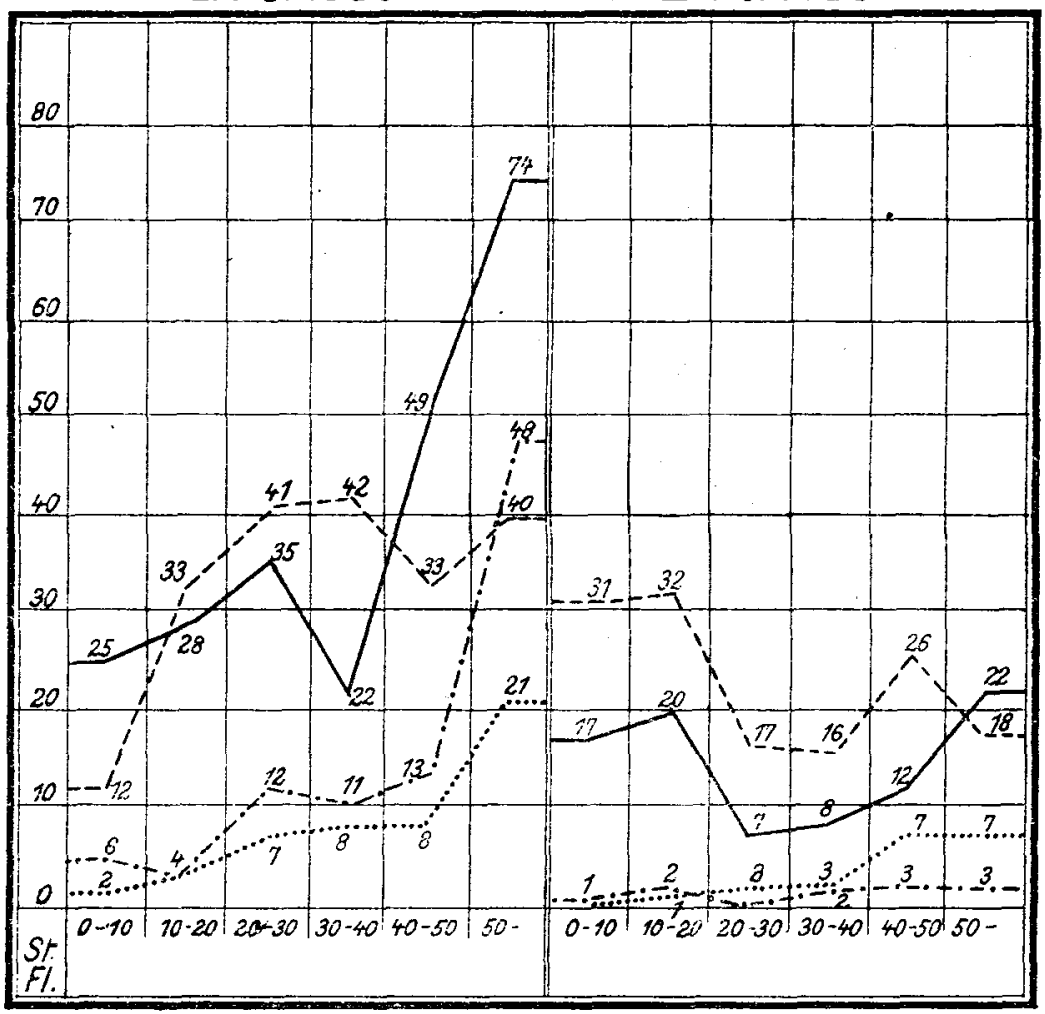

Morbiditat der Flüchtlinge

-.... " "Siädter

-..... Mortalität der Flüchtlinge

........." " "Städter

Fig. 3.

Mit aller Klarheit geht das Gesetz aus den Prozentzahlen hervor. 
Es sterben

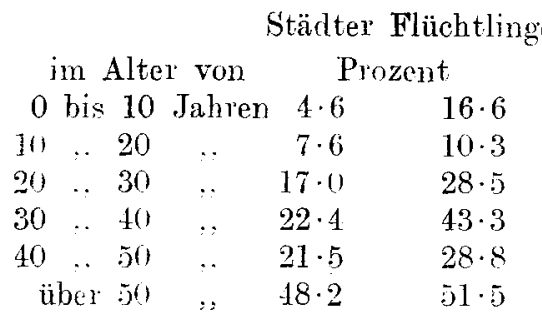

Daß die Sterblichkeit der Flïchtlingskinder höher ist als die der Personen zwischen 10 und 20 Jahren. ist nicht als reine Wirkung der Krankheit anzuschen, sondern ist die Folge schlechter Wartung und Ernährling, die bei den Säuglingen und Kindern deletär wirkt, während sich ältere Individuen trotz mangelhafter Pflege durchsetzen.

Von etwa 671 Krankheitsfällen betreffen 400 das weiblicle. 271 das männliche Geschlecht. Zwischen 0und 20 Jahren differiert die Beteiligung nur gering. Von 20 bis 40 Jahren uiberwiegen die Frauen un fast 50 Prozent, und zwar deshalb, weil die Mämner im militärpflichtigen Alter nach der Moldau abgerïckt sind: bei Frauen über 40 Jahren sinkt der Interschied gegenüber dem männlichen Element herab.

im Alter von Männlich Weiblich

$\begin{array}{rrrrr}0 \text { bis } 10 & \text { Jahren } & 38 & 47 \\ 10 \quad, 20 & 20 & 58 & 55 \\ 20 . . & 30 & . & 30 & 61 \\ 30 . . & 40 & . & 30 & 58 \\ 40 .: 50 & . & 43 & 79 \\ \text { uiber } 50 \quad ., & 53 & 81\end{array}$

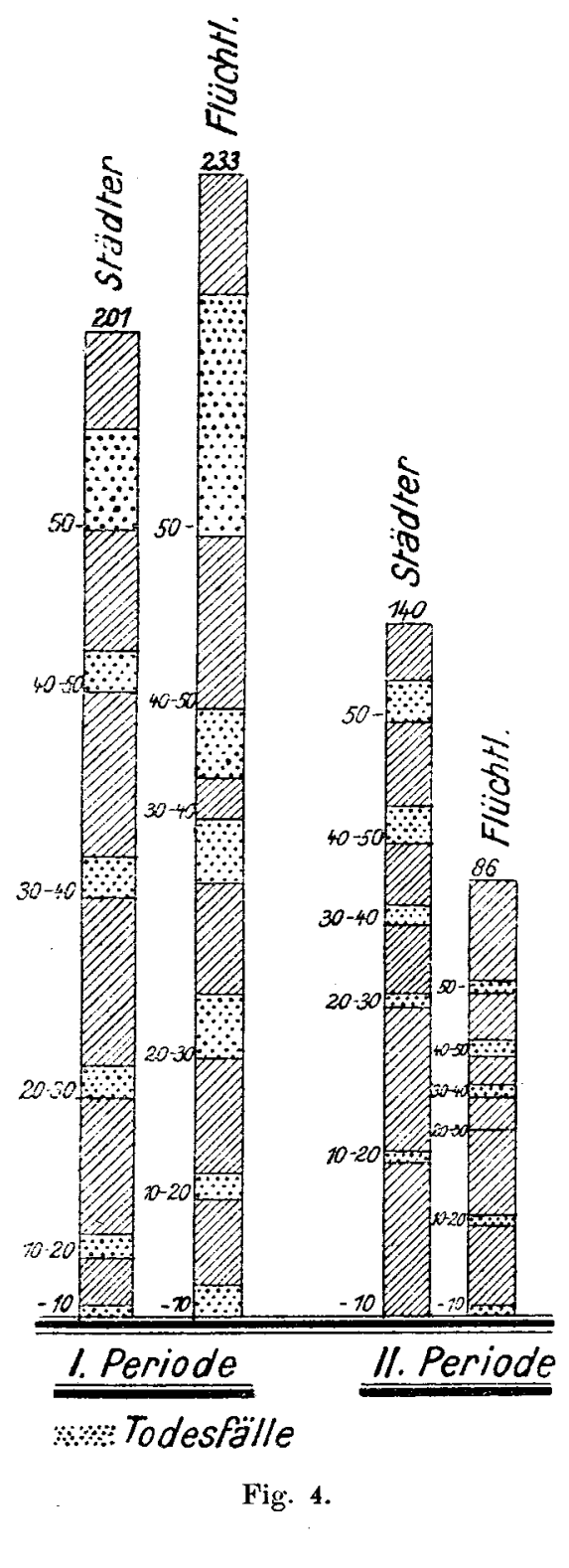

Auf der Höle der ersten Epidemieperiode im Juni und Juli ist die erhöhte Anteilnahme des weiblichen Geschlechts an der Morbidität besonders auffällig. 


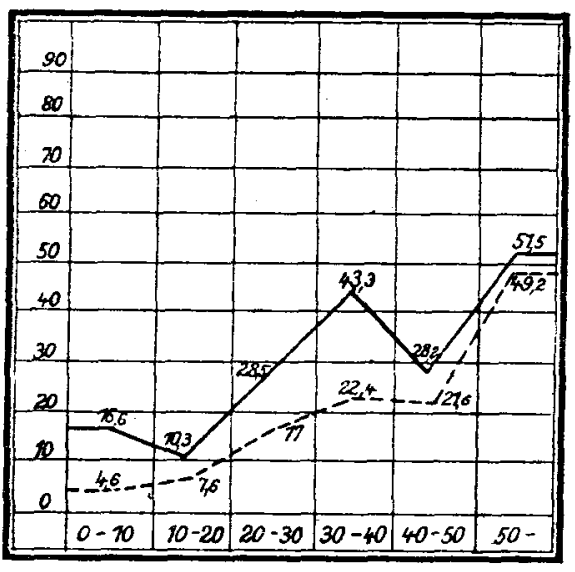

flüchtlinge
Städter
März . . . 13

April . . . . 28

Mai . . . 43

Juni . . . . . . 8

Juli . . . . 12

August . . . . 11

September . . $t$

Oktober . : . 4

November . . 11

Dezember . . 18

Januar . . . 43

Februar . . 24
Weiblich

8

48

15

95

.57

17

\section{4}

8

14

28

53

22

Von 400 weiblichen Individuen starben $74=18$ Prozent.

Von 271 männlichen starben $111=40.9$ Prozent.

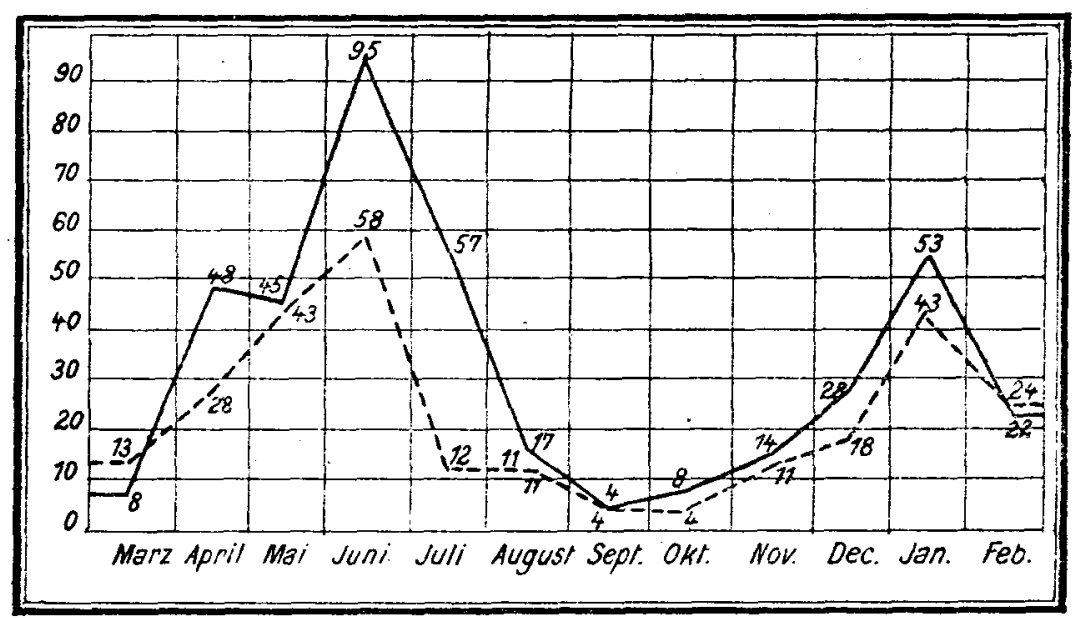

Männlich.

Weiblich.

Fig. 6.

Es sterben:

Männlich

im Alter von 0 bis 10 Jahren 4

$\begin{array}{lllllll}\text {.. } & 10 & . . & 20 & \text {.. } & 0\end{array}$

$\begin{array}{llllll}. & 20 & . & 30 & . & 7\end{array}$

$\begin{array}{lllllll}. . & 30 & . & 40 & . . & 7\end{array}$

$\begin{array}{lllll}. . & 40 & : 50 \quad, 5 & 14\end{array}$

$\begin{array}{lllll}. . & \text { iiber } 50 & \ldots & 42 & 79 \cdot 2\end{array}$

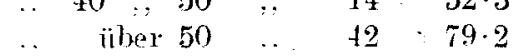

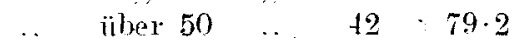

$10 \cdot 5$ Prozent

$6: 10 \cdot 3$

$7: 23 \cdot 3$

$7 \quad: 23 \cdot 3$

$32 \cdot 3$ 
Von 53 Männern über 50 Jahren starben $42=79 \cdot 2$ Prozent, während von 84 Frauen im gleichen Alter $37=44$ Prozent zugrunde gingen.

Im Mai und Juni ist die Sterblichkeit der männlichen Bevölkerung am höchsten. Im Mai starben von 43 Männern $18=41 \cdot 8$ Prozent; im Juni starben von 58 Männem $25==43 \cdot 1$ Prozent.

Im Zusammenhang mit der Gesamtmortalität stellt die Fleckfiebersterblichkeit nur einen kleinen Koeffizienten dar. Von den 4159 Personen, die innerhalb des Beobachtungsjahres verstorben sind, wird nur bei 177 als Todesursache Typhus exantlematicus angegeben, nur bei 4.2 Prozent.

Mortalität an Fleckfieber korrespondiert nicht einmal mit der allgemeinen Sterblichkeit, demn der Monat August mit des grausamen Sterblichkeitsziffer von $778 \mathrm{Per}$ sonen verzeichnet nur 11 Todesfälle an Typhus exanthematicus.

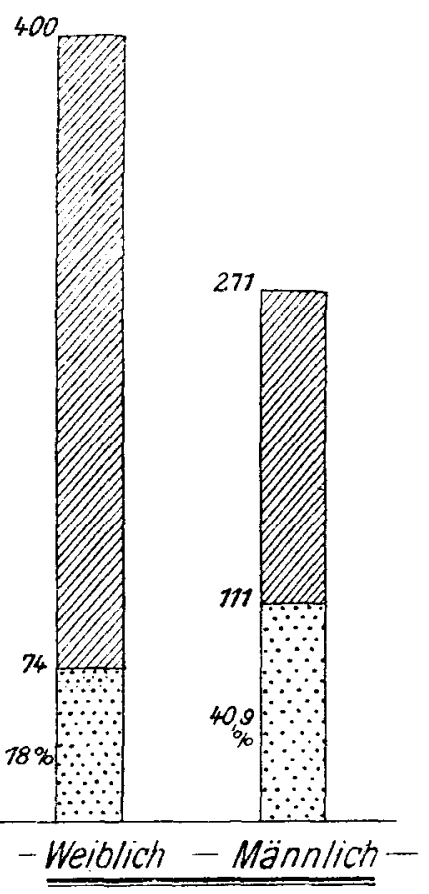

Fig. 7 .

Männ/ich.

Weiblich

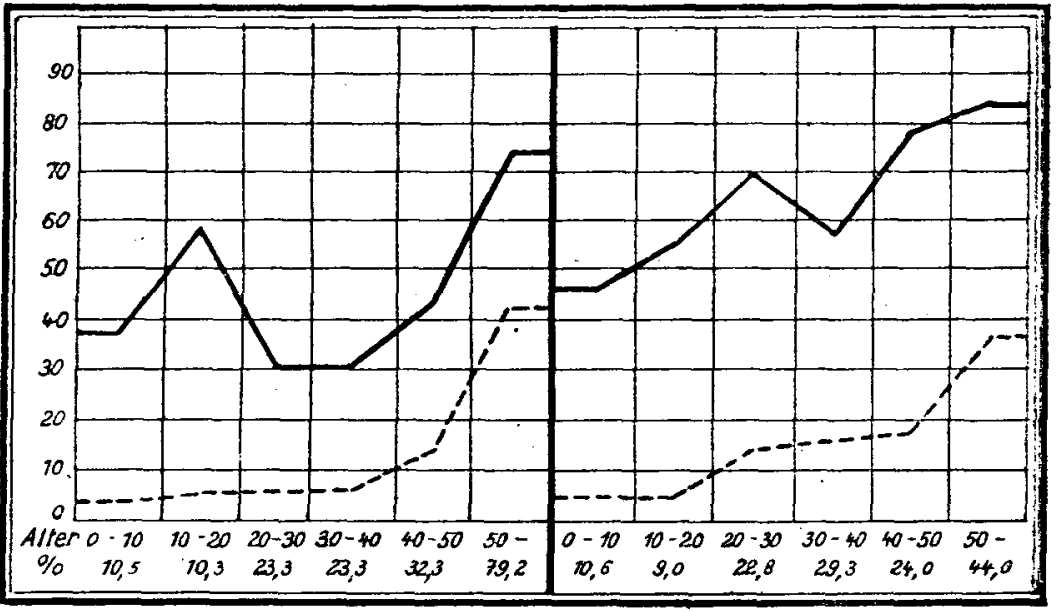




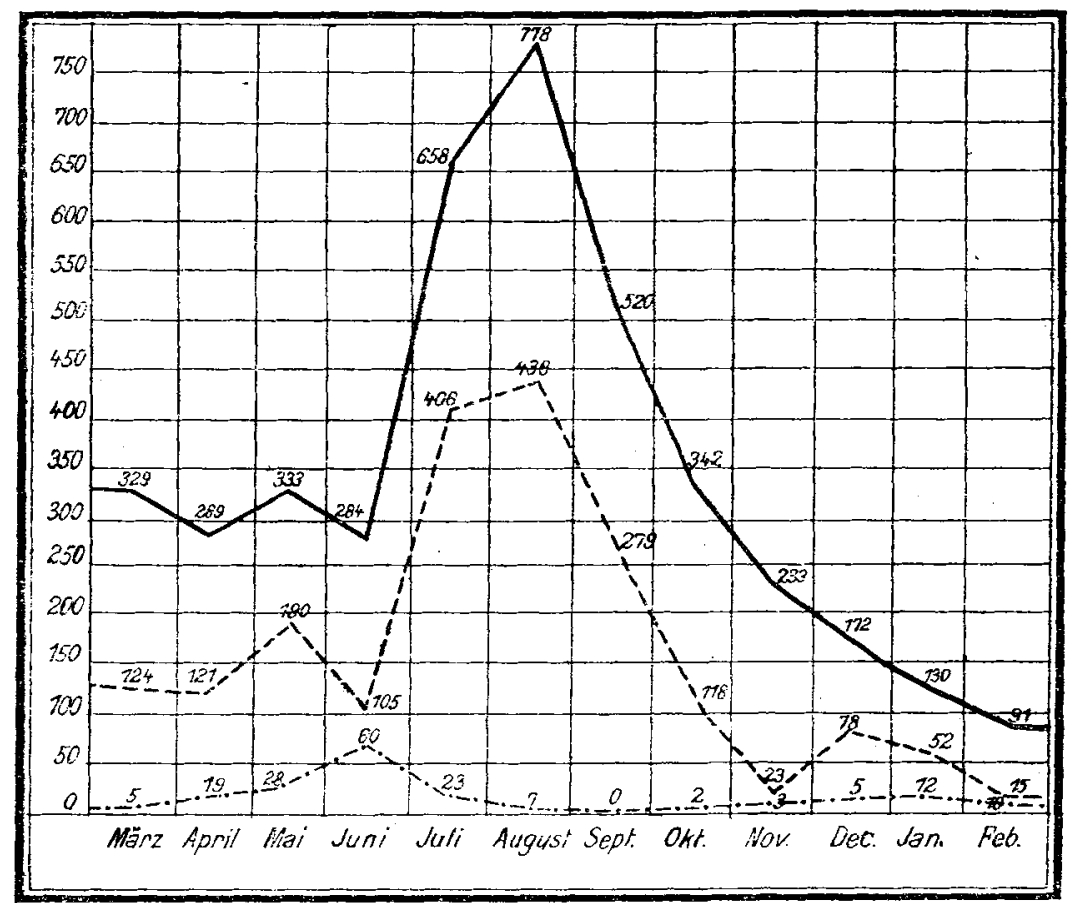

\section{Gesamtsterblichkeit der Städter.}

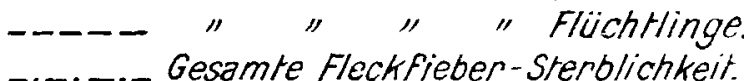

Fig. 9.

Zu den Pränissen Wohnungsnot und Nahrungsmittelmangel trat als fördernder Faktor ein Ereignis, das, als hygienische Vorsichtsmaßregel gedacht, paradox wirkte und eine ungeahnte Verbritung der Fleckfiebererkrankung zur Folge hatte: die Typhussehutzimpfung der Bevölkernng Foceanis.

In April und Mai wurden durchschnittlich am Tag 2 bis 3 Fleckfieberfälle verzeichnet.

Am 22. Mai begannen die Impfungen Ċer Zivilbevölkerung. Genau 15 Tage nach Beginn der Impfung, am 6. Juni 1917, stieg die Zacke auf 15 Fälle an.

Dieses Phänomen wurde mit der Impfung in Zusammenhang gebracht. Die Tatsache, daß durch die Impfung die Fleckfieberkurve sehr ungünstig beeinflußst wurde, wird durch folgende Erwägungen ursächlich erklärt:

1. Die massierte Ansammlung von mangelhaft entlausten Menschen in an Impfbureaus bedingte einen innigen Kontakt und dadurch eine 
Übertragung von Läusen. Die Ủbertỵgung wurde dadurch begïnstigt, daß die Personen zur Impfung ihre Oberkleider ablegen miBten. So wurden die Impfbureaus gefährliche Infektionsterde, die das Fleckfieber weit und schnell verbreiteten.

2. Jede Impfung bedeutet einen Fingriff in den Organismus, den gut genährte Individuen leicht übersteken; scllecht genährte werden durch diesen Akt so geschwächt, daB ihr Widerstand gegeniiber den Infektionserregen sehr herabgeseizt wird.

Die Inpfung wirkte auf cie Bewohner von Focsani so ungïnstig. dab sie in großer Zahl der Fleckficberinfektion unterlagen und erkrankten.

3. Durch die Typhusschutzimpfung wird der Organismus zur Bildung von Antikörpern gegen das Typhusgift stark angeregt. Der Sckeinangriff des Typhusabdominalis-Bazillus löst eine so allgemeine Reaktion des Körpers aus, daß er keine Truppen gegen den wahren Feind, den Fleckfiebererreger, ins Treffen führen kann und ohne Kampf die Waffen streckt. Die hohe Infektiosität des Fleckfiebererregers im Juni lag also an der Disposition der Individuen, die zugleich die schlechte Prognose des Trphus exanthematicus in jenem Zeitabschnitt verursachte.

Am 8. Juni wurde durch Kommandanturbefehl die Impfung ausgesetzt.

Erst nach einem Monat, im Juli, beruhigte sich die plötzlich aufgestörte Epidemie, die durch cinen Sturm anfgeworfene Welle, die so manches Lebensschifflein verschlungen hat.

Die epidemiologischen Lehren aus der hier bearbeiteten Fleckfieberepidemie sind folgende:

1. In Focsani hat es Typhus exanthematicus vor dem Kriege nicht gegeben.

2. Wohnungsnot und Nahrungsmittelnot prädisponieren die Epidemie.

3. Zwischen Winter- und Sommerepidemie gibt es eine Kontinuität, dargestellt durch leichte Fälle.

4. Der prognostische Charakter der Epidemie korrespondiert mit der allgemeinen Lage der Bevölkerung.

5. Die Malignität des Typhus exantlematicus wächst mit dem Lebensalter.

6a. Das weibliche Element ist stärker an der Epidemie beteiligt als das männliche.

6b. Das Fleckfieber der Frauen hat eine bessere Prognose als das der Männer.

7. Durch allgemeine Typhusschutzimpfungen wird die Fleckfieberepidemie ungünstig beeinflußt. 


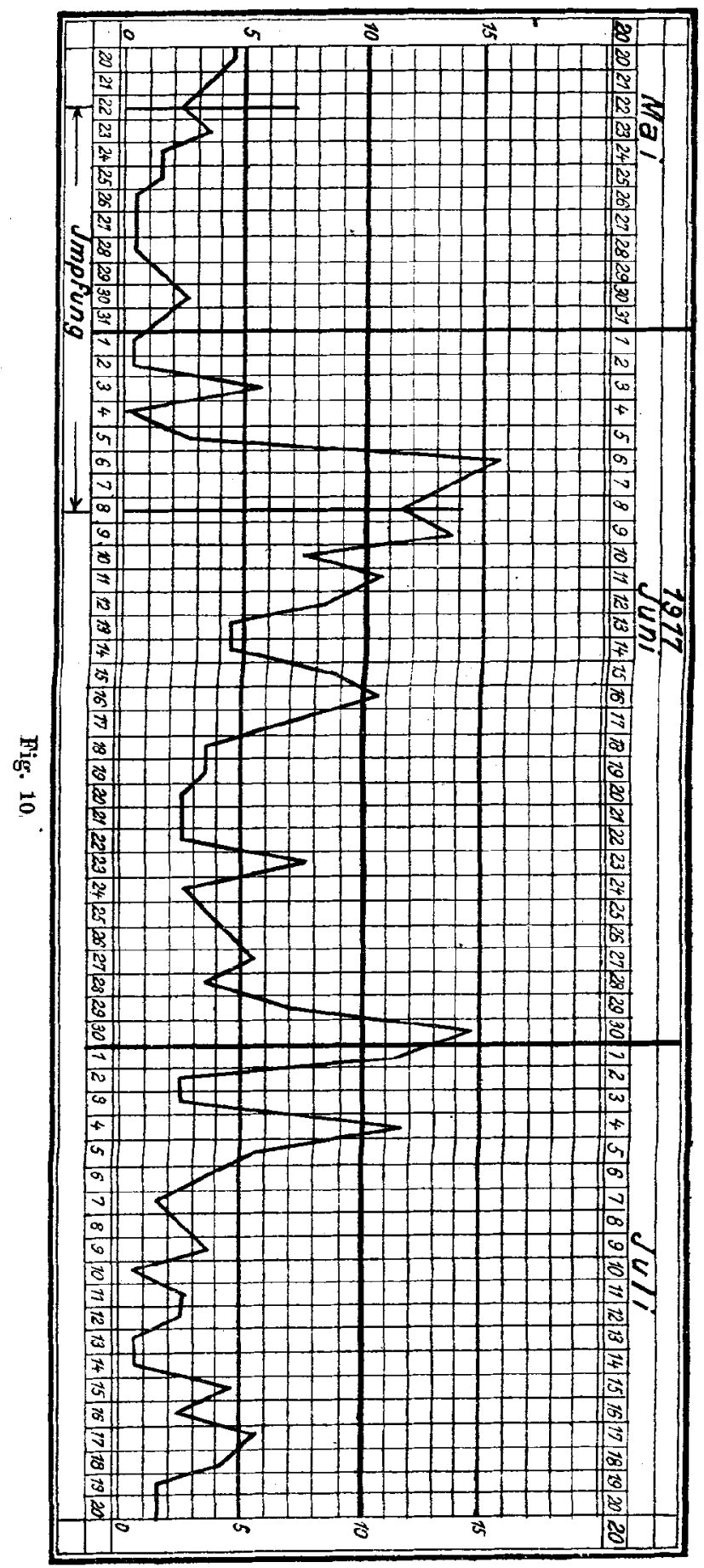


Die Maßnahmen gegen die Fleckfieberepidemie konnten in Anbetracht der kriegerischen Verhältnisse nicht sofort systematisch in idealer Vollendung ergriffen werden, sondern entwickelten sich aus improvisierten, primitiven Anfängen im Laufe der Zeit. Schon die Einführung einer geordneten Verwaltung nach den kriegerischen Wirren wirkte günstig auf die hygienische Lage der Stadt.

Als erste allgemeine hygienische Einrichtung wurce die Volksernährungsstelle gesctaffen. Ihre Aufgabe besteht in der Erfassung der vorhandenen Nahrungsmittel und ihrer Rationierung. Sie erwirkt den Nachschub aus militärischen Beständen und systematisiert unter Ausschaltung des freien Handels die Verteilung. Aus den Erträgen werden Volksküchen für die Armen eingerielitet und Untersützung bis 40000 Lei monatlich an bedürftige Personen verabreiclit. Das Verhältnis von Volksernälrung zur Epidemie ist aus den Fleckfieberkurven ersichtlich: die Lebensmittelversorgung besserte sich nach der Ernte im August 1917. Seit dieser Zeit verliert die Seuche an Kraft; im September treten nur sporadische Fälle mit 0 Prozent Mortalität auf. Die kältere Jałreszeit bringt wieder eine relative Nahrungsmittelnot, die sich im Anstieg der Seuchenkurve symbolisiert.

Dic Erkenntnis, daß die Seuckenherde in den Fliebtlingsquartieren -gelegen waren und daß von dort die Epidemie über die Stadt kam, schuf am 24. Juni 1917 die Flüchtlingskommandantur, eine militärische Dienststelle, die eine Verbesserung der Lage der Flüchtlinge erstrebte.

Die Flüchtlinge wurden nach Dorfgemeinden zusammengelegt, und es wurden die Verwaltungsorgane, welche im Frieden in den Dörfern funktionierten, wieder eingesetzt, die itre Geneinden nach Instruktionen der Flüchtlingskommandantur leiteten.

Die Ordnung der Verhältnisse hatte günstige Wirkungen:

1. Die Quartiernot wurde durch eine rationellere Verteilung vermindert.

2. Durch Einrichtung von Volkskücl en für Flüchtlinge besserte sich der Ernährungszustand.

3. Die wirtschaftlichen Interessen, die bisher ohne Anwalt dastanden, wurden wahrgenommen.

Diese umfassenden Maßnahmen hoben entschieden den Gesundkeitszustand der Flüchtlinge, und cler Nieçergang der Seucłenkurve im Juli und in den folgenden Monaten ist besonders anf diese soziale Anlage zurückzuführen.

Doch lieb sich trotz aller Bemühungen eine wesentlicke Bessorung nicht erreiclen, weshalb der Abtransport der Fliichtlinge in ein gliicklickeres Hinterland betrieben wurde. 
Es wurden al)transportient:

tarben

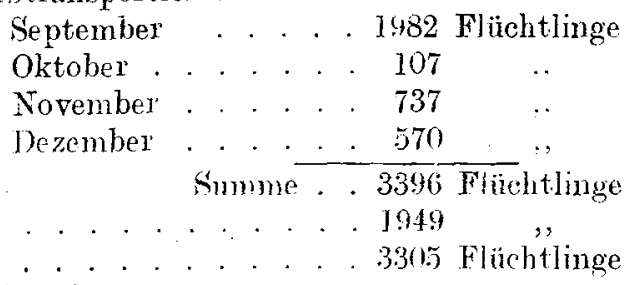

in Focsani zur ückblieben.

Erst durch das schwierige, ins Leben der Fiuchtlinge tief eingreifente Unternehmen des Abtransportes wurde eine relative Sanierung durchgesetzt. Die Fluichtlingsfürsorge hatte am 12. Januar 1918 ihre Aufgabe erfüllt; die Flüchtlingskommandantur wurde aufgekoben.

Wie ein Schlag traf die Fleckfieberepidemie die Bevölkerung. ohne daß sie gegen diesen furchtbaren Feind gerüstet war.

Die Mobilisienung der Kampfmittel begann mit der Reorganisation des durch den Krieg völlig aufgelösten rumäniselen Sanitätswesens, die vom Garnisonserzt durchgeführt wurde.

Die rumänischen Ärzte und das rumänische Sanitätspersonal werden in den Dienst der Gesundheitspflege der Stadt gestellt. Die Stadt wird in vier Sanitätsbezirke eingeteilt mit je einem rumäniscten Arzt an der Spitze, der die Fenktionen des Armen- und Seuchenarztes ausuibt.

J.-No. . .

den

An Garnisonarzt

Meldung des Dr. . . . . . .

Name:

Strada:

Nr. ....

Alter:

Temp.

Diagnose :

Seit wann krank .... Tge. Militär?...

Arbeiter-Abt.?

Spitalsbedürftig:

Bemerkungen:
Anordnungendes Garnisonarzte:

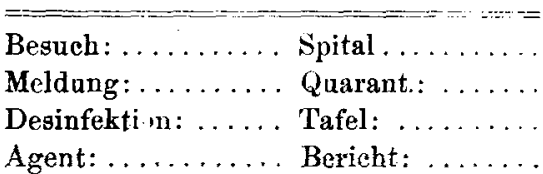

Bericht II : Dat: .... Diagnosn: .... Temp.: ..... Bemerkungen:

Dr. .

\section{Anordnungen :}

Bericht III : Dat:.... Diagnose: .... Temp.: ..... Bemerkungen: .......

Dr. .............

Anordnungen :

A BerichtIV: Dat: .... Diagnose: .... Temp.: ..... Bemerkuogen:

Dr. ............. 


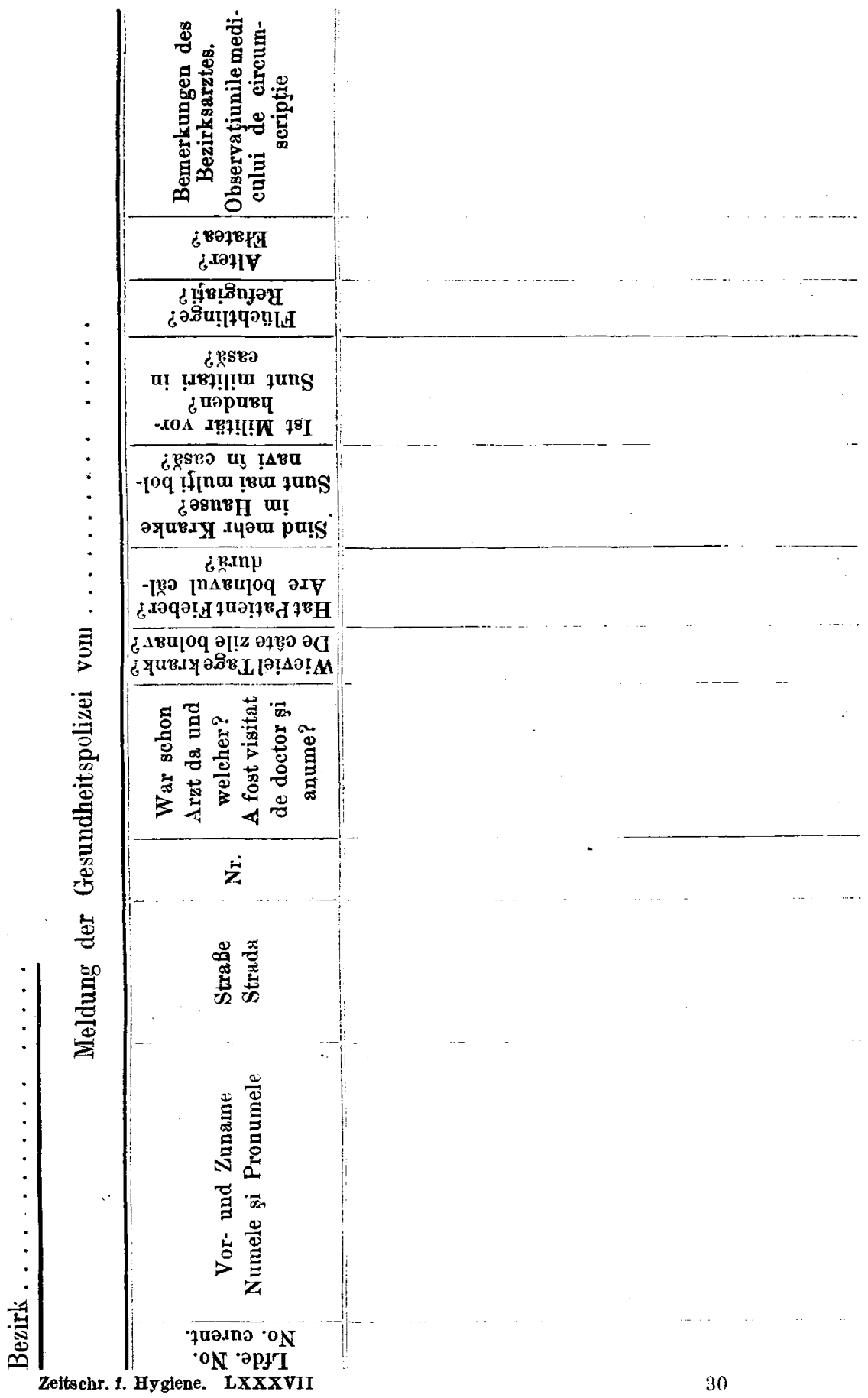


Es ist ein Meldezwang von Infektionskrankheiten eingeführt auf Formularen, die nach bestimmten Gesichtspunkten abgefaßt sind (s. S. 464).

Beamte der Sanitätspolizei sucben regelmäßig die Stadt ab, um ansteckende Krankheiten, die dem Bezirksarzt nicht bekanṇt geworden sind, zu erfassen. Sie melden auf Formularen, die dem Bezirksarzt zur Feststellung der Diagnose weitergeleitet werden (s. S. 465).

Die Krankenhäuser aus rumänischer Zeit werden, soweit sie nicht von deutschen Lazaretten belegt sind, wieder in Betrieb genommen.

Ein rumänisches Krankenhaus wird als Seuchenspital eingerichtet.

Eine Quarantänestation für Angehörige von Fleckfieberkranken ist im Norden der Stadt in Verbindung mit einer Entlausungsanstalt gebaut worden.

Zwei Desinfektionstrupps, die unter Führung je eines deutschen Soldaten stehen, sind gebildet; sie setzen sich aus fleckfieberfesten Rumänen zusammen. Die sichere und schnelle Bekämpfung der Läuse wird das oberste Prinzip. Die Läusevertilgung wird in erster Linie mit einer physikalisch wirksamen Methode vorgenommen: mit strömendem. Wasserdampf, der in wenigen Minuten Läuse und Nissen mit Sicherheit abtötet. Strömender Wasserdampf wird in fabrbaren Desinfektionskarren erzeugt. Die Abtötung der Läuse wird auch chemisch geuibt. Zur zuverlässigen Entlausung von Wohnräumen hat sich die Schwefelung bewährt, am besten durch Verbrennung von einem Schwefelkohlenstoffgemisch (90 Gewichtsprozente Schwefelkohlenstoff, 5 Gewichtsprozente Wasser, 5 Gewichtsprozente denaturierten Spiritus), im Handel fertig unter dem Namen Salfarcose erhältlich. Die Schwefeldämpfe müssen in vorgeschriebener Konzentration 6 Stunden eịnwirken.

Eine Zivilbade- und Entlausungsanstalt wird, um eine Entlausung der Stadt durchzuführen, in großem Ma ßstab angelegt. Die Anlage ist so getroffen, daß Eingang und Ausgang in verschiedenen Straßen liegen. So wird eine scharfe Trennung zwischen reiner und unreiner Seite gewährleistet.

Die Entlausung wird mit Heißluft von 80 bis $100^{\circ}$ in zwei Öfen vorgenommen. Sie sind nach dem System der Backöfen gebaut. Die Innentemperatur wird durch Naphthalometer zur Kontrolle. regelmäßig nachgeprüft. In 20 Minuten sind bei einer Temperatur von $100^{\circ}$ sämtliche Läuse und Nissen getötet.

Zur körperlichen Reinigung wird ein Brausebad, zu dem eine SeifenSodalösung geliefert wird, verabreicht. Bei besonders starker Verlausung wird das Kopfhaar kurz geschoren, Scham- und Achselhöhlenhaare werden mit Strontiumsulfidpaste entfernt. 
Es baden im Durchschnitt täglich 800 Personen; die maximale Leistungsfähigkeit der Anstalt beträgt 1500 Personen am Tag.

Die hygienische Behandlung eines Fleckfieberfalles gestaltet sich folgendermaßen:

1. Erfassung des Falles durch den Garnisonarzt mit Hilfe des rumänischen Arztes und der Sanitätspolizei.

2. Sofortige Spitalisierung des Fleckfieberkranken.

3. Isolierung der Angehörigen in der Quarantänestation.

4. Sehließung des Fleckfieberquartiers.

5. Bezeichnung des Fleckfieberquartiers durch eine Seuchentafel.

Haus geschlossen.

Bewohner in Quarantäne.
Casa închisă. Locatarii în Carantină.
A Ansteckende Krankheiten.

Boală

Molipsitoare Eintritt verboten. Intrarea Oprita.

Der Orts-Kommandant.

6. Nach 18 Tagen Desinfektion des Quartiers und dreimalige Wiederholung derselben in achttägigen Zwischenräumen.

Das Militär wird grundsätzlich in Flüchtlingsbezirken nicht einquartiert. Befindet sich im Hause eines Fleckfieberkranken eine Militärperson, so wird sie sofort nach Meldung ausquartiert, entlaust und unter Beobachtung gestellt.

Unter diesen nach und nach einsetzenden sozialen, wirtschaftlichen und hygienischen Maßnahmen wurde die Fleckfieberziffer kontinuierlich herabgesetzt und vor allem erreicht, daB die Epidemie nicht auf das deutsche Heer übergegangen ist. 